\title{
A one-dimensional model of viscous blood flow in an elastic vessel
}

Fredrik Berntsson, Matts Karlsson, Vladimir Kozlov and Sergey A. Nazarov

\section{Linköping University Post Print}

\section{Tweet}

N.B.: When citing this work, cite the original article.

Original Publication:

Fredrik Berntsson, Matts Karlsson, Vladimir Kozlov and Sergey A. Nazarov, A onedimensional model of viscous blood flow in an elastic vessel, 2016, Applied Mathematics and Computation, (274), 125-132.

http://dx.doi.org/10.1016/j.amc.2015.10.077

Copyright: Elsevier

$\underline{\text { http://www.elsevier.com/ }}$

Postprint available at: Linköping University Electronic Press

http://urn.kb.se/resolve?urn=urn:nbn:se:liu:diva-124453 


\title{
A one-dimensional model of viscous blood flow in an elastic vessel
}

\author{
Fredrik Berntsson * Matts Karlsson* Vladimir Kozlov* \\ Sergey A. Nazarov ${ }^{\dagger}$
}

March 30, 2015

\begin{abstract}
In this paper we present a one-dimensional model of blood flow in a vessel segment with an elastic wall consisting of several anisotropic layers. The model involves two variables: the radial displacement of the vessel's wall and the pressure, and consists of two coupled equations of parabolic and hyperbolic type. Numerical simulations on a straight segment of a blood vessel demonstrate that the model can produce realistic flow fields that may appear under normal conditions in healthy blood vessels; as well as flow that could appear during abnormal conditions. In particular we show that weakening of the elastic properties of the wall may provoke a reverse blood flow in the vessel.
\end{abstract}

\section{Introduction}

Blood vessels form one of the most complicated and important systems in the human body. The system is exposed to various risks and is poorly amenable to medical treatments. A wide variety of diseases and ailments are caused by disturbances in the blood circulatory system. This makes understanding the system important; and mathematical models of different levels of complexity useful.

The existing one-dimensional models are based on three relations accepted a priori: conservation of fluid mass and momentum together with a linear stationary tube law connecting the cross-section of the tube with the pressure. As a result, after an intrinsic linearization, one obtains a hyperbolic type equation with respect to pressure; or another longitudinal variable. Such models are described in details in $[4,13,22,24,25]$, where one can find a more detailed analysis and references to miscellaneous variants of such models.

In contrast to the existing one-dimensional models, see [26], our model, rigorously derived in [7]-[10] by means of asymptotic analysis and a dimension reduction procedure, leads to a hyperbolic-parabolic type system of differential

\footnotetext{
*Linköping University, SE-58183 Linköping, Sweden. Emails: fredrik.berntsson@liu.se, matts.karlsson@liu.se, and vladimir.kozlov@liu.se

†St Petersburg State University, St Petersburg State Polytechnical University, and Institute of Problems of Mechanical Engineering RAS, Russia. Email: srgnazarov@yahoo.co.uk
} 

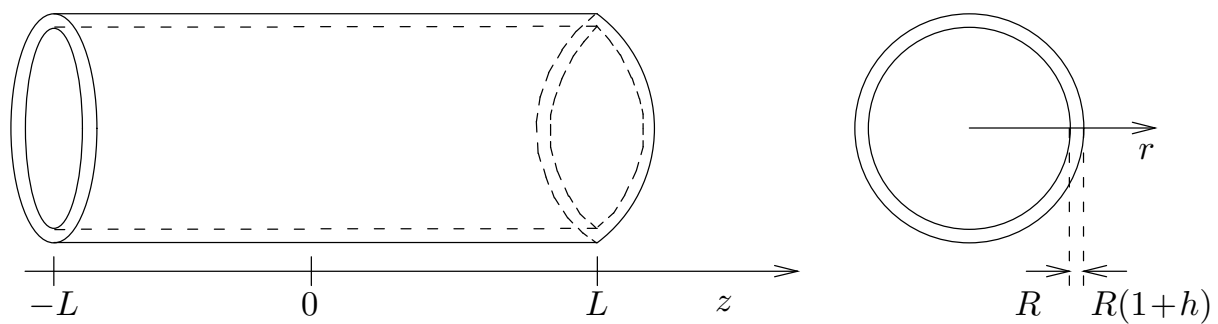

Figure 1: The geometry of the blood vessel. The thickness of the vessel wall $h$ is small compared to the radius $R$ and the length $2 L$.

equations. The model includes an analysis of the kinematic interaction between the elastic walls and the blood flow, and, e.g., makes the above-mentioned traditional tube law non-local and non-stationary.

In our work we focus on the blood flow in a single artery. The goals are twofold. First, to present a simple (one-dimensional, linear) model of a straight segment of an artery, which takes into account the elastic properties of vessels wall, but neglect the surrounding muscle tissue, cf. [7, 8]. This model is based on modern techniques from asymptotic analysis and contain as a particular case the standard one dimensional model. Second, to find physical parameters of the system which can provide a reverse blood flow (in the direction to the heart). A reverse flow contradicts the purpose of the artery and does not happen during normal conditions. A reverse flow can be caused, for example, by weakening the elastic properties of the vessel due to aging of the collagen fibers or dissection of the wall. The point is that the system of differential equations serving as the mathematical model for the blood flow in the vessel segment under consideration represents a coupling of a hyperbolic and a parabolic equation. These equations work in discord, and therefore the model can reproduce a wide range of flow regimes. If, e.g, a disease provokes a mismatch in the physical parameters the system can be brought out of the normal working regime and mimic flow behavior that occur for patients under influence of cardiovascular diseases. In particular, a reversal of the blood flow in the aorta, can happen in patients suffering from cardiac dysrhythmia, or arrhythmia.

This paper is organized as follows: In Section 2 we present our mathematical model, for details of a derivation of this model we refer to [7]-[10]. In Section 3 we describe the numerical implementation, and also present results from two test simulations that demonstrate that our model reproduces realistic flow patterns. Finally in Section 4 we give some conclusions and discuss the future development of the model.

\section{A Mathematical Model of Blood Flow}

In this section we develop a mathematical model that describes a single cylindrical shaped blood vessel with a circular cross-section. We try to reduce the complexity of the model to the nessecary minimum, that still supports new effects, and take into account only the principal terms in the asymptotic expansions of the physical fields. Although the dimension reduction procedure used 
also gives us expressions for the higher order terms, which can lead to models of increased accuracy, our goal of finding physical conditions where a reverse flow appears is achived within the simple model. Let

$$
\Theta_{h}^{R}=\{x=(y, z): r=|y|<R(1+h), z \in(-L, L)\}
$$

be a circular cylinder consisting of a thin elastic wall,

$$
\Sigma_{h}^{R}=\Theta_{h}^{R} \backslash \Theta_{0}^{R},
$$

and the interior of the vessel ${ }^{1} \Theta_{0}^{R}$. The dimensionless parameters $h$ and $\delta=R / L$ are supposed to be small and $h<<\delta$. The surface where the blood flow interacts with the wall of the vessel is denoted by

$$
\Gamma_{0}^{R}=\{x: r=R, z \in(-L, L)\} .
$$

The domain is illustrated in Figure 1.

The blood flow is assumed to be symmetric around the $z$-axis. The physical fields we are interested in are the velocity vector $\mathbf{v}=\left(v_{r}, v_{z}\right)^{T}$ for the blood flow inside the vessel, the displacement vector for the vessel's wall $\mathbf{u}=\left(u_{r}, u_{z}\right)^{T}$, and the pressure $p$ of the blood. Within the Euler approach and under the conditions of small deformations in the wall as well as a relatively slow flow of blood, see Section 2.1, we reduce all constitutive relations to the reference surface (5), where $R$ denotes a constant typical radius, while the actual radius of the vessel clearance is $R+u_{r}(z, t)$.

The velocity and the pressure satisfy the Navier-Stokes system:

$$
\left\{\begin{aligned}
\gamma_{b}\left(\partial_{t} v_{r}+v_{r} \partial_{r} v_{r}+v_{z} \partial_{z} v_{r}\right) & =\mu\left(\Delta v_{r}-r^{-2} v_{r}\right)-\partial_{r} p \\
\gamma_{b}\left(\partial_{t} v_{z}+v_{r} \partial_{r} v_{z}+v_{z} \partial_{z} v_{z}\right) & =\mu \Delta v_{z}-\partial_{z} p
\end{aligned}\right.
$$

and the continuity equation,

$$
\partial_{r} v_{r}+r^{-1} v_{r}+\partial_{z} v_{z}=0,
$$

where we used a cylindrical coordinate system, $\Delta=\partial_{r}^{2}+r^{-1} \partial_{r}+\partial_{z}^{2}$ is the Laplace operator, $\gamma_{b}$ is the density of the blood, and $\mu$ is the dynamical viscosity coefficient of the blood. The dynamical non-slipping condition:

$$
\mathbf{v}=\partial_{t} \mathbf{u}, \quad \text { on } \Gamma_{0}^{R},
$$

where $\partial_{t} \mathbf{u}$ represents the velocity of the wall's displacement, is imposed on the surface where the blood flow interacts with the vessel's wall.

The wall of the blood vessel consists of three layers. Two of them contain collagen fibers with different winding angles and are filled with weaker cell material, see $[3,6]$. In $[9,10]$ the procedure of dimension reduction was applied and the following intuitively clear result was obtained rigorously: the thin composite shell interacting with the flow of liquid is modeled by an orthotropic (with the main axes $r, \phi$ and $z$ ) elastic surface $\Gamma_{0}^{R}$. In other words, due to $z$-axis symmetry of all physical fields the following limit system is valid:

$$
\left\{\begin{array}{l}
h\left(k_{\varphi \varphi} \varepsilon_{\varphi \varphi}+k_{\varphi z} \varepsilon_{z z}\right)+h R \gamma_{w} \partial_{t}^{2} u_{r}+f_{r}=0 \\
-h R \partial_{z}\left(k_{z \varphi} \varepsilon_{\varphi \varphi}+k_{z z} \varepsilon_{z z}\right)+h R \gamma_{w} \partial_{t}^{2} u_{z}+f_{z}=0
\end{array}\right.
$$

\footnotetext{
${ }^{1}$ In [10] it was proved that a circular form of the cross-section is optimal, see also [9].
} 
Here $u_{r}, u_{z}$ and $\varepsilon_{\varphi \varphi}=R^{-1} u_{r}, \varepsilon_{z z}=\partial_{z} u_{z}$ are components of the displacement vector and deformation tensor in cylindrical coordinates; $k_{\varphi \varphi}, k_{\varphi z}=k_{z \varphi}$ and $k_{z z}$ are the effective elasticity modulus of the vessel's wall, $\gamma_{w}$ is the density of the vessel's wall, and $f_{r}$ and $f_{z}$ are force per unit area. The above formulas mean a cancellation of the interior tension in the shell, the inertial forces caused by its oscillations, and the external forces from liquid's flow ${ }^{2}$.

Although the rigorous asymptotic procedure of homogenization of the triliminar elastic wall, armored with collagenic fibres, is rather complicated, see $[18,20]$ and $[8,9]$, the resulting equations $(7)$ have a simple mechanical interpretation: the sum of the active forces vanishes. Reading from right to left in (7) these forces are hydrodnamic, inertial and elastic in the longitudinal and circumferical directions. The latter are computed through the orthotropic linear Hooke's law.

Finally, the hydrodynamic forces are given by the following formulas:

$$
f_{r}=-p+\mu \partial_{r} v_{r}, \quad f_{z}=\frac{\mu}{2}\left(\partial_{z} v_{r}+\partial_{r} v_{z}\right) .
$$

\subsection{Scaling analysis}

First we assume that the relative thickness $h>0$ of the blood vessel's wall, cf. Figure 1, is small and also that the relative radius $\delta=R / L$ is small. Introduce a time-like parameter,

$$
\tau=\delta^{2} t
$$

and define the Reynolds and Stokes numbers as,

$$
\operatorname{Re}=\frac{\gamma_{b}}{\mu} \frac{1}{T} \delta L^{2} \text { and } \mathrm{St}=\frac{\gamma_{b}}{\mu} \frac{1}{T} \delta^{2} L^{2}=\delta \operatorname{Re} .
$$

Since the relative radius $\delta<<1$ we conclude that the Reynolds number is also relatively small, which guarantees a laminar, and slowly varying, blood flow. This allows us to exclude the inertial and convective terms from the NavierStokes system (the left-hand sides in the equations (4)). The quantity $T$ is a characteristic length of time (e.g. the reciprocal of the pulse).

We also introduce two Womersley numbers, one for the fluid and another for the elastic properties of the vessel:

$$
\mathrm{Wb}=(\mathrm{St})^{1 / 2} \text { and } \mathrm{W} \mathrm{w}=\frac{R}{T}\left(\frac{\gamma_{w}}{k_{\varphi \varphi}}\right)^{1 / 2},
$$

where $\gamma_{w}$ is the effective density of the wall. The first Womersly number is small due to the assumption of smallness of the numbers (10). The second Womersley number depends on many factors, including the physical properties of the blood vessel under consideration. For veins, or peripheral vessels, the number $W_{w}$ will be small, partly due to an increase of the characteristic length of time $T$, and also due to a decrease in the density of collagen fibers in the walls. Dealing with healthy arteries, we assume that Ww has order one when deriving the limit system of equations, but in numerical experiments we shall vary it in a certain range.

\footnotetext{
${ }^{2}$ The effects of transmural pressure, orthotropy and viscoelasticity of the walls has been studied by different methods in $[28,27,23,11,12]$
} 


\subsection{Dimension reduction}

In the previous section we discussed the relative magnitude of the parameters included in the model. Since the relative thickness $h$ of the blood vessel's wall, the relative radius $\delta$, and the time-like parameter $\tau$ are small we can use the ansatz:

$$
\begin{aligned}
v_{z}(r, z, \tau) & =\frac{1}{4 \mu}\left(r^{2}-R^{2}\right) \partial_{z} \bar{p}(z, \tau)+\delta v_{z}^{\prime}(r, z, \tau)+\ldots, \\
v_{r}(r, z, \tau) & =\delta \cdot 0+\delta^{2} v_{\tau}^{\prime}(r, z, \tau)+\ldots \\
p(r, z, \tau) & =\bar{p}(z, \tau)+\delta p^{\prime}(r, z, \tau)+\ldots
\end{aligned}
$$

which means that we have the Poiseuille flow as the leading term. The ansatz means that equation (4), with zero right-hand sides, is satisfied up to higher order terms. For explicit expressions for the correction terms $v_{z}^{\prime}, v_{\tau}^{\prime}$, and $p^{\prime}$, see [19]. Furthermore, the continuity equation (5) together with the non-slipping condition (6), where

$$
\begin{aligned}
& u_{z}(z, \tau)=0+\delta u_{z}^{\prime}(z, \tau)+\ldots \\
& u_{r}(z, \tau)=\delta u_{r}^{0}(z, \tau)+\delta^{2} u_{r}^{\prime}(z, \tau)+\ldots,
\end{aligned}
$$

lead to the one-dimensional Reynolds equation,

$$
\partial_{\tau} u_{r}^{0}(z, \tau)-\frac{L^{3}}{16 \mu} \partial_{z}^{2} \bar{p}(z, \tau)=0 .
$$

Due to (12), the hydrodynamical forces (8) take the form

$$
f_{r}(r, z, \tau)=-\bar{p}(z, \tau)+\delta f_{z}^{\prime}(r, z, \tau)+\ldots, \quad f_{z}(r, z, \tau)=0+\delta f_{z}^{\prime}(r, z, \tau)+\ldots
$$

Now keeping the main asymptotic terms only and taking into account (14), the second equation in system (7) is satisfied up to lower order terms and the first one can be simplified as

$$
\frac{h}{L} k_{\varphi \varphi} u_{r}^{0}(z, \tau)+h R \delta^{5} \gamma_{w} \partial_{\tau}^{2} u_{r}^{0}(z, \tau)=\bar{p}(z, \tau),
$$

Introducing new variable $\bar{u}_{r}=\delta u_{r}^{0}$ and returning to the variable $t$, we write equations (14) and (15) as

$$
\begin{aligned}
& h R^{-1} k_{\varphi \varphi} \bar{u}_{r}(z, t)+h R \gamma_{w} \partial_{t}^{2} \bar{u}_{r}(z, t)=\bar{p}(z, t), \\
& \partial_{t} \bar{u}_{r}(z, t)-\frac{R^{3}}{16 \mu} \partial_{z}^{2} \bar{p}(z, t)=0 .
\end{aligned}
$$

We note that the asymptotic analysis from [8], see also [7], shows that taking into account the impact of external muscle reaction on the vessel leads to a decrease of the effective elasticity modulus $k_{\varphi \varphi}$.

\subsection{The parameters of the one-dimensional model}

In this section we discuss the relation between the parameter $k_{\varphi \varphi}$ in (16) and the rest of the parameters included in the mathematical model discussed in Section 2 .

The parameter $k_{\varphi \varphi}$ represents an average elasticity modulus. We recall that the walls of the blood vessel consists of three layers with different mechanical 
and structural properties. The intima is a thin interior layer which does not influence the elastic properties of the vessel. The remaining two layers are the media and the adventicia, see [6]. Both these layers consists of a loose cell material strengthened by a regularly ordered mesh of collagen fibers. The fibers in the two different layers have winding angles $\varphi_{m}$ and $\varphi_{a}$ with respect to the symmetry axis of the blood vessel.

The homogenization procedure of plates and shells with sharply contrasting strengthening fibers (see [18] and [10]) gives the following value to the coefficient $k_{\varphi \varphi}$ in (7):

$$
h k_{\varphi \varphi}=2 E\left(\sin ^{4} \varphi_{m}+\sin ^{4} \varphi_{a}\right),
$$

where $E$ is the Young's modulus of the collagen fibers. We note that the elastic modulus with respect to longitudinal tension, which can be found experimentally, take the form

$$
h k_{z z}=2 E\left(\cos ^{4} \varphi_{m}+\cos ^{4} \varphi_{a}\right),
$$

i.e. quantity (18) and (19) can be recalculated in terms of each other.

Observe, that formulas (18) and (19) are valid for healthy vessels only; see details in [10]. For example, in the case of dissection of a vessel's wall, media and adventia loose contact and a certain load vector is applied to the cell material of the vessel, which drastically change the elastic properties of the vessel's wall. Conversely, calsious, i.e. precipitation of calcium salts on the interior vessel's wall, makes the wall more fragile. These examples demonstrate that the range of realistic values for the module of elasticity $k_{\varphi \varphi}$ may be very large.

\section{$3 \quad$ Numerical implementation}

In this section we discuss our numerical implementation for solving the equations of our model. Numerical simulations of blood flow in veins or arteries have been carried out by several authors, see e.g. [17, 15, 1, 2, 16]. In contrast our mathematical model can be solved with a simple and very efficient numerical method.

As previously discussed the asymptotic analysis leads to two equations to solve. First the pressure $\bar{p}=\bar{p}(z, t)$ and the radial component of the wall displacement $\bar{u}_{r}=\bar{u}_{r}(z, t)$ satisfies

$$
\bar{p}(z, t)=K \bar{u}_{r}(z, t)+G \partial_{t}^{2} \bar{u}_{r}(z, t), \quad 0 \leq t \leq T,-L<z<L,
$$

where,

$$
K=h R^{-1} k_{\varphi \varphi}, \quad \text { and } \quad G=h R \gamma_{w},
$$

and

$$
\partial_{t} \bar{u}_{r}(z, t)=\frac{R^{3}}{16 \mu} \partial_{z}^{2} \bar{p}(z, t), \quad 0 \leq t \leq T,-L<z<L .
$$

Since both $\bar{p}$ and $\bar{u}_{r}$ are periodic in $t$, with period $T$, it is natural to use a Fourier series representation,

$$
\widehat{p}\left(z, \xi_{k}\right)=\frac{1}{\sqrt{T}} \int_{0}^{T} \bar{p}(z, t) \mathrm{e}^{i \xi_{k} t} d t, \quad \xi_{k}=\frac{2 \pi k}{T}, k \in \mathbb{Z} .
$$


Inserting (21) into (20) we arrive at an equation for the Fourier coefficients $\hat{p}\left(z, \xi_{k}\right)$ of the pressure $\bar{p}$ in the vessel,

$$
\widehat{p}\left(z, \xi_{k}\right)=\frac{i R^{3}}{16 \mu}\left(G \xi_{k}-K \xi_{k}^{-1}\right) \partial_{z}^{2} \widehat{p}\left(z, \xi_{k}\right), \quad-L<z<L, k \in \mathbb{Z} .
$$

For the case of frequency $\xi_{k}=0$ we instead obtain the simpler equation $\partial_{z}^{2} \widehat{p}=0$, so that for this case the pressure is a first degree polynomial in $z$. Also for the case $K-\xi_{k}^{2} G=0$ we simply set $\widehat{p}\left(z, \xi_{k}\right)=0$, see (20). Since the equation (22) is of second order two boundary conditions are needed. In this paper we use,

$$
\partial_{z} \bar{p}(-L, t)=-\Phi(t), \Phi(t)=\frac{8 \mu}{R^{2}} \bar{v}_{z}(-L, t) \quad 0 \leq t<T,
$$

which means that we specify the mean flow velocity of the blood at $z=-L$. At the point $z=L$ we use the Dirichlet condition,

$$
\bar{p}(L, t)=p_{*}(t), \quad 0 \leq \tau<T .
$$

Having a Dirichlet condition at $z=L$ means that we have a well-posed problem for the zero frequency component $\hat{p}(z, 0)$, and we can recover the mean pressure.

In our implementation we select an equidistant grid $\left\{z_{i}\right\}_{i=1}^{N}$, and $\left\{t_{j}\right\}_{j=0}^{M}$, such that $z_{1}=-L, z_{N}=L, t_{0}=0$, and $t_{M}=T$. Note that since we have periodic boundary conditions in $t$ the points $t_{0}$ and $t_{M}$ are the same. The equation (22) is solved, for each frequency $\xi_{k}$ separately, using the standard finite difference approximation,

$$
\partial_{z}^{2} \hat{p}\left(z_{i}, \xi_{k}\right) \approx\left(\hat{p}\left(z_{i+1}, \xi_{k}\right)-2 \hat{p}\left(z_{i}, \xi_{k}\right)+\hat{p}\left(z_{i-1}, \xi_{k}\right)\right)(\Delta z)^{-2} .
$$

The Neumann condition (23) is implemented using a one-sided difference

$$
\partial_{z} \hat{p}\left(z_{1}, \xi_{k}\right) \approx\left(-3 \hat{p}\left(z_{1}, \xi_{k}\right)+4 \hat{p}\left(z_{2}, \xi_{k}\right)-\hat{p}\left(z_{3}, \xi_{k}\right)\right)(2 \Delta z)^{-1} .
$$

These approximations mean that the finite difference method is $\mathcal{O}\left(\Delta z^{2}\right)$ accurate. Note also that the resulting linear system of equations is tri-diagonal and can be solved very efficiently.

Remark 3.1. The proper choice of boundary conditions to is of course dependent which quantities that can be accurately measured at the respective boundaries $z=-L$ and $z=L$. In the case where measurements of the mean flow velocity $\bar{v}_{z}$ at the boundaries are available we differentiate the equation (22) with respect to $z$ and obtain,

$$
\widehat{v}_{z}\left(z, \xi_{k}\right)=\frac{i R^{3}}{16 \mu}\left(G \xi_{k}-K \xi_{k}^{-1}\right) \partial_{z}^{2} \widehat{v}_{z}\left(z, \xi_{k}\right), \quad-L<z<L, k \in \mathbb{Z},
$$

thus we can solve a Dirichlet problem, with the same equation, for the mean flow velocity $\bar{v}_{z}$ in the vessel.

Remark 3.2. Once the pressure field $\bar{p}$ is computed we obtain the radial displacement field $\bar{u}_{r}$ using equation (20), i.e.

$$
\widehat{u}_{r}\left(x, \xi_{k}\right)=\left(K-\xi_{k}^{2} G\right)^{-1} \widehat{p}\left(z, \xi_{k}\right) .
$$




\begin{tabular}{|l|l|l|l|l|}
\hline Physical Quantity & Symbol & Unit & Test 1 & Test 2 \\
\hline \hline Density of wall & $\gamma_{w}$ & $\mathrm{~kg} / \mathrm{m}^{3}$ & 1050 & 1450 \\
Viscosity of blood & $\mu$ & $\mathrm{kg} / \mathrm{m} \mathrm{s}$ & $5.1 \cdot 10^{-3}$ & $6.1 \cdot 10^{-3}$ \\
Radius of vessel & $R$ & $\mathrm{~m}$ & $6.3 \cdot 10^{-3}$ & $12.1 \cdot 10^{-3}$ \\
Relative thickness of wall & $h$ & & 0.15 & 0.78 \\
Modulus of Elasticity & $k_{\varphi \varphi}$ & $\mathrm{Pa}$ & $4.15 \cdot 10^{5}$ & $2.1 \cdot 10^{3}$ \\
Period in time & $T$ & $\mathrm{~s}$ & 0.917 & 0.43 \\
\hline
\end{tabular}

Table 1: The physical parameters that were used for our tests. For Test 1 we use values that roughly correspond to the carotid artery of a healthy individual. The values used for Test 2 are not entirely realistic and are used for the purpose of illustrating a reverse flow case.

\subsection{Numerical Experiments}

In order to demonstrate that our model works well in practice, and can produce realistic solutions, we give the results from two numerical simulations. ${ }^{3}$ As a basis for our calculations we use typical physical parameters that roughly correspond to the carotid artery for a healthy individual; see [21]. Also, the flow velocity profile used in the boundary condition (23) were taken from [5], where an experimental velocity profile was presented. For both simulations we pick an equidistant grid consisting of $N=500$ grid points in the $z$-variable, and $M=512$ points in the $t$-variable. This means that we attempt to recover frequency components in the range $\xi_{k}=2 \pi k T^{-1}$, for $-255<k<256$.

Test 1. Consider the blood flow in the carotid artery under normal conditions, as specified in Table 1 . We use the above mentioned experimentally obtained mean velocity profile for the boundary condition $\partial_{z} \bar{p}(-L, t)=-\Phi(t)$. At the far end of the vessel use a constant pressure $\bar{p}(L, t)=p_{*}$, where $p_{*}=80 \mathrm{mmHg}$, or $10.7 k P a$. The equations $(20)$ and $(21)$ are linear and we can set any zero level for the pressure; in our calculations we let $R=6.31 \mathrm{~mm}$ be the typical radius of the blood vessel when the pressure is at $11.5 \mathrm{kPa}$. Hence the wall displacement $\bar{u}_{r}$ can be both positive and negative. In the simulations $L=1 \mathrm{~m}$; but we only present the results in the interval $\left[-L,-L+2 L_{0}\right]$, for $L_{0}=10 \mathrm{~cm}$. The numerical results are presented in Figure 2. While the boundary condition $\bar{p}(L, t)=p_{*}$ is not entirely realistic both the pressure field and flow velocity near the boundary $z=-L$ demonstrates that the model can produce realistic solutions.

Remark 3.3. For the parameters used in Test 1 we calculate the constants of (22) as:

$$
K=7.84 \cdot 10^{6}, \quad G=7.88 \cdot 10^{-2} .
$$

This means that under normal conditions in a healthy individual the inertia term $G \partial_{t}^{2} \bar{u}_{r}$ in (20) can be neglected leading to a simpler one-dimensional model. In this case our model is similar to other $1 D$ models previously reported in the literature, see for instance $[14,24]$, and also [22]. Neglecting the inertia term

\footnotetext{
${ }^{3}$ In [8] there is also an analytic solution that has been used to verify the numerical procedure.
} 

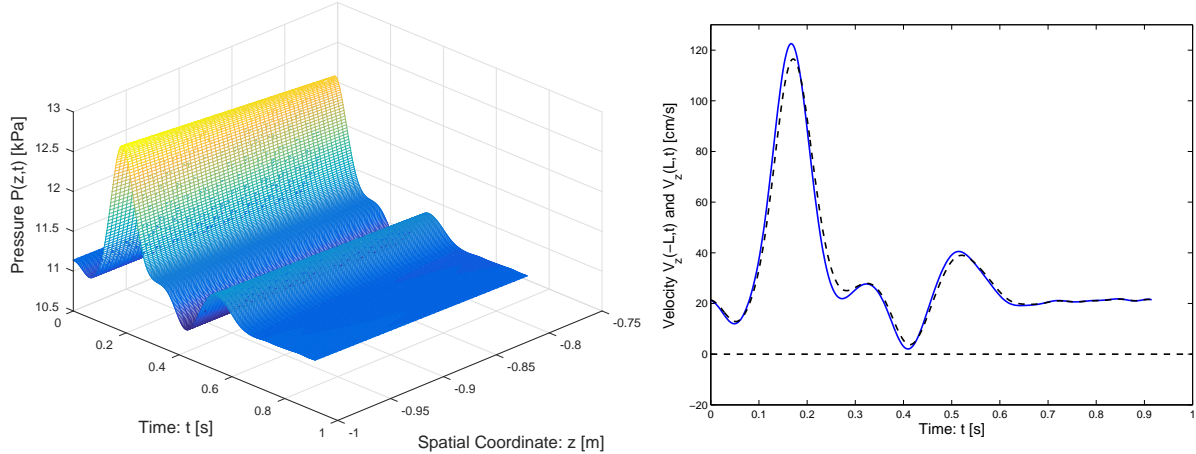

Figure 2: The computed pressure $\bar{p}(z, t)$ for the case of a normal healthy carotid artery (left), and also the experimental mean flow velocity profile $\bar{v}_{z}(-L, t)$ used for the boundary condition at $z=-L$ (right,blue solid curve) and the computed velocity profile $\bar{v}_{z} 20 \mathrm{~cm}$ into the vessel (right,black dashed curve). We remark that the flow velocity at $z=-L$ is strictly positive, and that the flow velocity stays strictly positive in the entire vessel segment $[-L, L]$

also gives us an approximate linear relationship between pressure $p$ and wall displacement $\bar{u}_{r}$ as

$$
\bar{p}=h R^{-1} k_{\varphi \varphi} \bar{u}_{r}
$$

For our test a pressure in the range of $10.7-12.6 \mathrm{kPa}$ results in a wall displacement of magnitude $0.13 \mathrm{~mm}$. The numerical relationship obtained from our simulation is displayed in Figure 4.
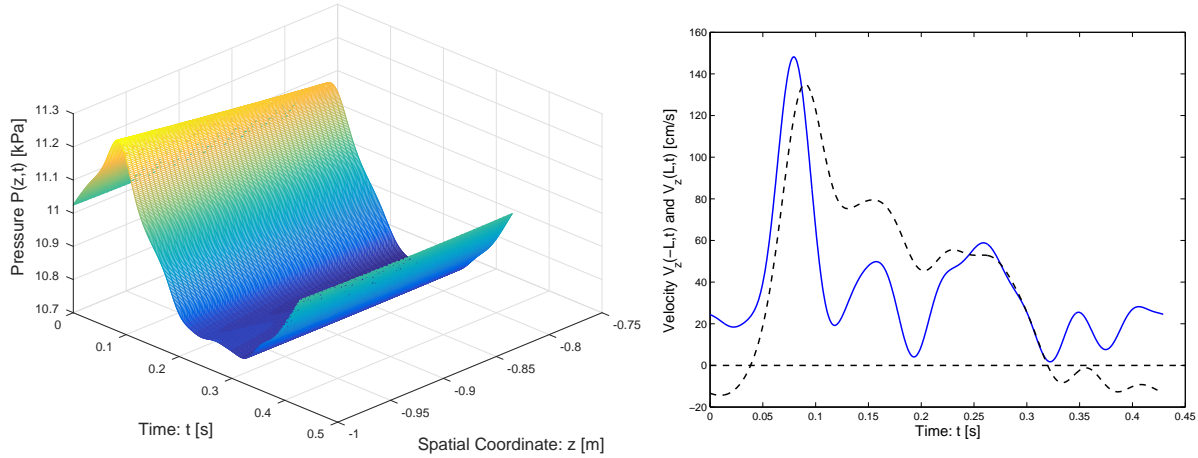

Figure 3: The computed pressure $\bar{p}(z, t)$, in the first $20 \mathrm{~cm}$ of the vessel, for a case where a reverse flow appears (left). Also the flow velocity profile $\left.\bar{v}_{z}\right|_{z=-L}$, obtained from the data $\Phi(t)$, used as a boundary condition at the inlet of the vessel (right,blue solid curve) and the computed velocity profile $\bar{v}_{z} 20 \mathrm{~cm}$ into the vessel (right,black dashed curve). We remark that the initial flow velocity is strictly positive and a reverse flow only appears inside the vessel. 
Test 2. In our second experiment we aim to make inertia term $G \partial_{t}^{2} \bar{u}_{r}$ in (20) more relevant. The constants used for this test were

$$
K=1.36 \cdot 10^{5}, \quad G=13.77 \cdot 10^{0} .
$$

In order to find a situation where a reverse flow appears we used the boundary conditions from Test 1 as a starting point and modified the functions $\Phi(t)$ and $p_{*}(t)$ randomly until a reverse flow was found. The results are displayed in Figure 3. Note that the inflow at $z=-L$ is strictly positive and the reverse flow only appears inside the vessel.

Note that this experiment does not correspond to a realistic patient. In order to emphasize the inertia term we reduced the characteristic period of time $T$ and increased the vessel's radius $R$. Also the module of elasticity $K_{\varphi \varphi}$ is lower; which could be due to broken collagen fibers, aging of the elastic material, or dissection, and the viscosity $\mu$ of blood is increased a little. In the case of broken collagen fibers much of the pressure on the artery is due to the surrounding muscle tissue; thus accounting for the increased relative thickness $h$ of the vessel's wall. The exact parameters used for this test is presented in Table 1.

In Figure 4 we display the relationship between the pressure $p$ and the wall displacement $\bar{u}_{r}$. For this experiment the inertia term $G \partial_{t}^{2} \bar{u}_{r}$ causes a small, but visible, hysteresis effect. The wall's position does not instantly adjust to a change in the pressure. At pressure $p=11.17 k P a$ the difference in $\bar{u}_{r}$ is about $0.06 \mathrm{~mm}$; for increasing and decreasing pressure respectively.
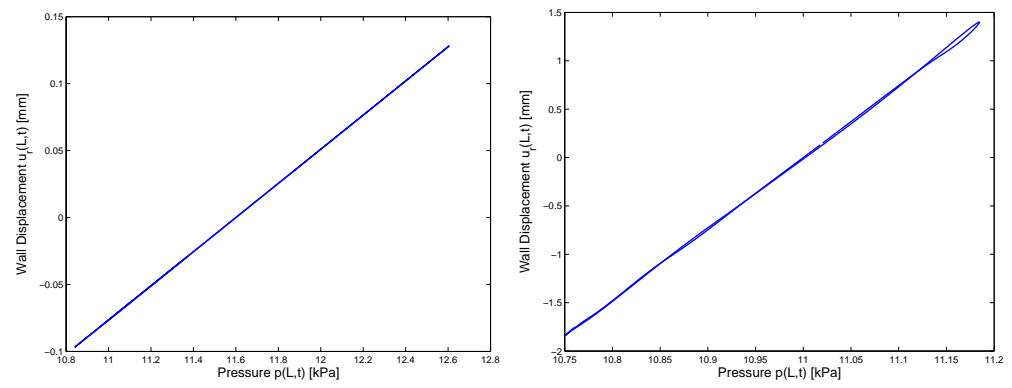

Figure 4: The wall's displacement $\bar{u}_{r}$ as a function of pressure $p$. In the case of a healthy artery (left) the relationship is linear, as expected from an elastic tube. For the reverse flow case (right) the displacement $\bar{u}_{r}$ is generally of larger magnitude due to the lower modulus of elasticity $k_{\varphi \varphi}$. In both cases the flow profile $20 \mathrm{~cm}$ into the vessel, i.e. at $z=-L+2 L_{0}$, was used to obtain the relationship between $p$ and $\bar{u}_{r}$.

\section{Concluding Remarks}

In this paper we have presented a simple linear one dimensional model for the blood flow in a single blood vessel. The model is derived from the Navier-Stokes equations and a model of the elastic properties of the walls of the vessel, using asymptotic analysis. 
Under normal conditions the model is similar to previously reported one dimensional models; however by changing the physical parameters of the flow we model behave more as a wave-equation, and our model can produce solutions that includes a reverse flow in the interior of the blood vessel under consideration. This means that our model can produce flow fields that appear both during normal conditions and under abnormal conditions that appear while under the influence of diseases.

The model is simple but there are several ways to make the model more realistic. First most of the parameters, e.g. the modulus of elasticity of the vessel's wall $K_{\varphi \varphi}$ or the relative thickness $h$, can be allowed to depend on $z$. For instance a more realistic reverse flow case could be constructed by altering the physical parameters in a small segment of the blood vessel. Also the curvature of the vessel should be taken into account. This is something we intend to do in the near future.

Further, in this work we study a single blood vessel. By deriving suitable boundary conditions to use at bifurcations we intend to extend the model to the entire arterial tree. Since the basic model is one dimensional, and the numerical solution technique is efficient, we expect that the numerical calculations for the entire tree can be carried out in reasonable time.

\section{References}

[1] V. Casulli, M. Dumbser, and E. F. Toro. Semi-implicit numerical modeling of axially symmetric flows in compliant arterial systems. Int. J. Numer. Meth. Biomed. Engng., 28:257-272, 2012.

[2] F. Fambri, M. Dumbser, and V. Casulli. An efficient semi-implicit method for three-dimensional non-hydrostatic flows in compliant arterial vessels. Int. J. Numer. Meth. Biomed. Engng., 30:1170-1198, 2014.

[3] Y.C. Fung. Biomechanics: mechanical properties of living tissues. SpringerVerlag, 1993.

[4] L. Grinberg, E. Cheever, T. Anor, J. R. Madsen, and G. E. Karniadakis. Modeling blood flow circulation in intracranial arterial networks: A comparative 3d/1d simulation study. Annals of biomedical engineering, 39(1):297-309, 2011.

[5] D W Holdsworth, C J D Norley, R Frayne, D A Steinman, and B K Rutt. Characterization of common carotid artery blood-flow waveforms in normal human subjects. Physiological Measurement, 20(3):219, 1999.

[6] G.A. Holzapfel. Collagen. Structure and Mechanic, chapter Ch. 11. Collagen in Arterial Walls: Biomechanical Aspects, pages 285-324. SpringerVerlag, 2008.

[7] V. A. Kozlov and S. A. Nazarov. An asymptotic model of the interaction of blood flow with vein walls and the surrounding muscle tissue. Dokl. Akad. Nauk, 446(6):631-636, 2012.

[8] V. A. Kozlov and S. A. Nazarov. Asymptotic models of blood flow in arteries and veins. Zap. Nauchn. Sem. S.-Peterburg. Otdel. Mat. Inst. 
Steklov. (POMI), 409(Matematicheskie Voprosy Teorii Rasprostraneniya Voln. 42):80-106, 242, 2012.

[9] Vladimir Kozlov and Sergei Nazarov. Surface enthalpy and elastic properties of blood vessels. Doklady physics, 56(11):560-566, 2011.

[10] Vladimir Kozlov and Sergei Nazarov. Asymptotic models of anisotropic heterogeneous elastic walls of blood vessels. submitted, 2014.

[11] G. D. C. Kuiken. Approximate dispersion equations for thinwall liquid filled tubes. Appl. Sci. Res., 41:37-53, 1984.

[12] G. D. C. Kuiken. Wave propagation in a thinwalled liquid filled initially stressed tube. J. Fluid Mech, 141:289-308, 1984.

[13] J. Lighthill. Mathematical biofluiddynamics. SIAM, 1975.

[14] Koen S. Matthys, Jordi Alastruey, Joaquim Peiró, Ashraf W. Khir, Patrick Segers, Pascal R. Verdonck, Kim H. Parker, and Spencer J. Sherwin. Pulse wave propagation in a model human arterial network: Assessment of 1-d numerical simulations against in vitro measurements. Journal of Biomechanics, 40(15):3476-3486, 2007.

[15] L. O. Mller and E. F. Toro. Well-balanced high-order solver for blood flow in networks of vessels with variable properties. Int. J. Numer. Meth. Biomed. Engng., 29:13881411, 2013.

[16] L. O. Mller and E. F. Toro. Enhanced global mathematical model for studying cerebral venous blood flow. Journal of Biomechanics, 47:3361$3372,2014$.

[17] Lucas O. Mller, Carlos Pars, and Eleuterio F. Toro. Well-balanced high-order numerical schemes for one-dimensional blood flow in vessels with varying mechanical properties. Journal of Computational Physics, 242(0):53-85, 2013.

[18] S. A. Nazarov, G. Kh. Svirs, and A. S. Slutskiu. Homogenization of a thin plate reinforced with periodic families of rigid rods. Mat. Sb., 202(8):41-80, 2011.

[19] S.A. Nazarov and K.I. Pileckas. Reynolds flow of a fluid in a thin threedimensional channel. Litovsk. mat. sbornik., 30(4):772-783, 1990. English transl.: Lithuanian Math. J. 1990. Vol. 30, No. 4., pp. 366-375.

[20] S.A. Nazarov, G. Sweers, and A. Slutskij. The bend rigidity of a thin plate enforced by periodic families of isolated rods. Prikl. Mat. Mekh., 74(3):441454, 2011. English transl.: J. Appl. Math. Mech. 2011. Vol. 74, No. 3. pp. $313-322$.

[21] Niema M Pahlevan and Morteza Gharib. Aortic wave dynamics and its influence on left ventricular workload. PLoS One, 6(8):e23106, 2011.

[22] Timothy J. Pedley. Mathematical modelling of arterial fluid dynamics. Journal of Engineering Mathematics, 47(3-4):419-444, 2003. 
[23] Alfio Quarteroni, Massimiliano Tuveri, and Alessandro Veneziani. Computational vascular fluid dynamics: problems, models and methods. Computing and Visualization in Science, 2(4):163-197, 2000.

[24] Philippe Reymond, Fabrice Merenda, Fabienne Perren, Daniel Rüfenacht, and Nikos Stergiopulos. Validation of a one-dimensional model of the systemic arterial tree. American Journal of Physiology - Heart and Circulatory Physiology, 297(1):H208-H222, 2009.

[25] Charles A. Taylor and Mary T. Draney. Experimental and computational methods in cardiovascular fluid mechanics. Annual Review of Fluid Mechanics, 36(1):197-231, 2004.

[26] Frans N. van de Vosse and Nikos Stergiopulos. Pulse wave propagation in the arterial tree. Annual Review of Fluid Mechanics, 43(1):467-499, 2011.

[27] Sunica ani and Eun Heui Kim. Mathematical analysis of the quasilinear effects in a hyperbolic model blood flow through compliant axi-symmetric vessels. Mathematical Methods in the Applied Sciences, 26(14):1161-1186, 2003.

[28] Sunica ani, Josip Tambaa, Giovanna Guidoboni, Andro Mikeli, Craig J. Hartley, and Doreen Rosenstrauch. Modeling viscoelastic behavior of arterial walls and their interaction with pulsatile blood flow. SIAM Journal on Applied Mathematics, 67(1):164-193, 2006. 